\title{
DIGITAL BEAMFORMING TECHNIQUES FOR MULTI-CHANNEL SYNTHETIC APERTURE RADAR
}

\author{
Marwan Younis, Felipe Queiroz de Almeida, Federica Bordoni, Paco López-Dekker, Gerhard Krieger
}

\author{
German Aerospace Center (DLR), Microwaves and Radar Institute
}

\begin{abstract}
SAR instruments with multiple transmit/receive channels allow for a variety of operation modes. The trade-space includes the instrument and antenna parameters designed to enable various operation modes. These are utilized to achieve a required SAR performance derived from the mission and user requirements. Finding a solution in this multi-dimensional trade space is not trivial and often involves the compromise of parameters. This paper addresses the topic of SAR instrument design tailored to a set of operation modes. It explains and compares the SAR techniques and gives the performance.
\end{abstract}

\section{INTRODUCTION}

In the last years a clear trend towards multi-digital-channel SAR systems has manifested itself. Examples are TerraSAR$\mathrm{X}$ (two channels as a by-product of the redundancy concept), RADARSAT-2 for ATI applications, ALOS-2 to increase the azimuth resolution. This step towards multi-channel SAR instruments marks a paradigm change as a consequence of research activity results in the last decade. The main innovative characteristic of forthcoming generations of SAR systems is the use of multiple elevation and/or azimuth receiver channels combined with digital beamforming (DBF) capability $[1,2,3]$. This allows for the synthesis of multiple or dynamic digital receiver beams. Further, multiple transmit channels are being suggested as an extension to DBF systems.

The virtue of Multi-Channel SAR is that it extends the dimensionality of the trade-space. This allows the conception of new systems which overcome the "fundamental limitation" of conventional SAR. A good example is the simultaneous High-azimuth-Resolution and Wide-Swath SAR also known as HRWS [4], which improves two performance parameters without sacrificing others $[5,6]$.

The aim of this paper is to introduce the trade-space of multi-channel SAR and show potential instrument designs. The approach is to introduce the system and performance parameters and show how they can be traded by virtue of system examples. The instrument description remains at a conceptual level to avoid irrelevant technical details.

\section{SYSTEM REQUIREMENTS AND TRADE-SPACE PARAMETERS}

The most relevant system and performance parameters for the L-band SAR considered in this paper are shown in Fig. 1. Here the system parameters basically describe the instrument both in terms of quantities fixed by the system design such as antenna dimensions, and parameters which can be altered during operation (e.g. the Pulse Repetition Frequency: PRF).

The system parameters will be altered for the various implementations considered later, however, to allow for a "fair" comparison (and to limit the trade space dimension), three system parameters will be fixed. As shown in Fig. 1 these are the center frequency, total average Tx power, and orbit height (resulting, for the chosen swath width of $400 \mathrm{~km}$ in an incidence angle range from $25^{\circ}$ to $45^{\circ}$ ).

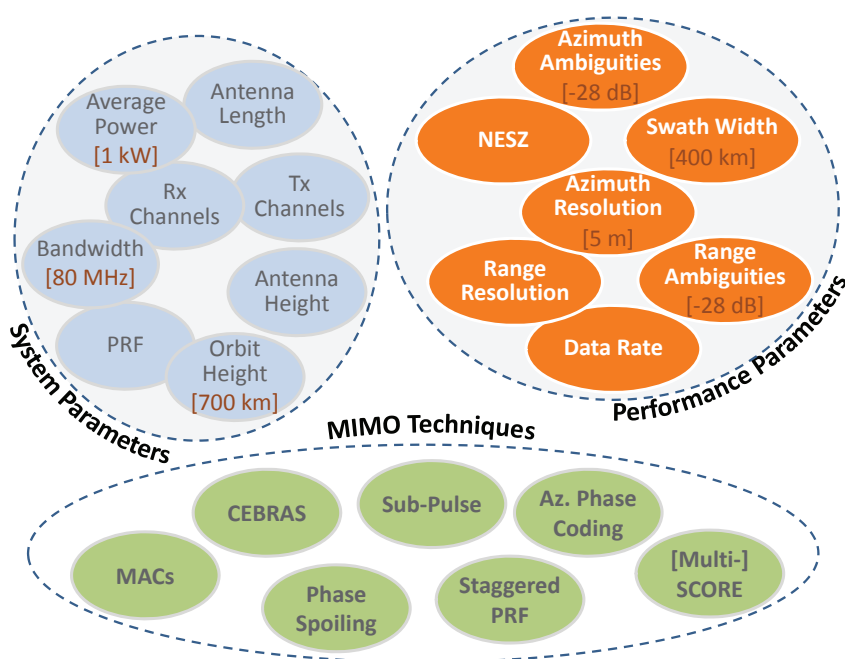

Fig. 1. Relevant system and performance parameters for SAR. These parameters can be optimized using different MIMO techniques (modes).

The performance parameters are used to describe how good the system actually is. The limiting values of a sub-set of the performance parameters are usually derived from the mission requirement, as is the case for the ambiguity level. 
Others may simply be a result of the system parameters, such as the range resolution assuming, here, that the chirp bandwidth is fixed. The actual values of the performance parameters result from a model- or simulation-based system analysis and obviously depend on the system parameters. To achieve the desired performance, various "MIMO" techniques might be used. Thus, for example the data rate (performance) depends on the system (chirp bandwidth) but also shows how efficiently the system is operated (techniques).

The emphasis of this paper is on the various SAR techniques, depicted in Fig. 1, that can be utilized for multichannel instruments to yield a desired performance.

\section{SAR SYSTEM OPTIONS}

In this section various SAR instrument and antenna options are investigated. The presented system designs should be understood as conceptional in the sense that further optimization would improve performance by several dBs. However, here the intention is to show how the various techniques can be utilized and to report on their system efficiency, which is a measure understood here as an indication of how efficiently the system resources are used to achieve the required performance.

\subsection{Single Swath Stripmap Mode}

A straightforward approach to reach the required swath width and azimuth resolution is shown in Fig. 2 and consists of dimensioning the Rx antenna so as to yield the swath width while utilizing Multiple Azimuth Channels (MACs) to reach the azimuth resolution. By this the resolution is decoupled from the total antenna length.

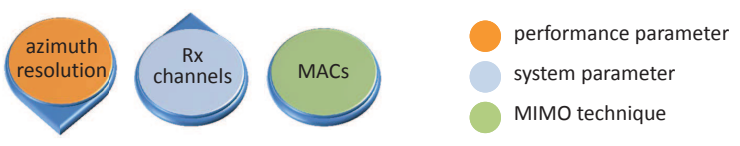

Fig. 2. Improving the azimuth resolution while maintaining the swath width by using MACs technique.

The system parameters are obtained by determining the $P R F$ which allows imaging a $W_{s w}=400 \mathrm{~km}$ swath. This gives $430 \mathrm{~Hz}$ when using the approximation

$$
P R F \leq \frac{c_{0}}{2 W_{s w} \sin \eta_{i}}
$$

where $\eta_{i}$ is the incidence angle and $c_{0}$ the velocity of light. The total antenna length is determined according to the condition of uniform azimuth sampling [2]

$$
L_{R x}^{\mathrm{unif}}=\frac{2 v_{\text {sat }}}{P R F}
$$

which yields a considerable value of $L_{R x}=34 \mathrm{~m}$ ! Knowing that the individual azimuth element must cover a Doppler bandwidth corresponding to the azimuth resolution (or just applying the known approximation for the azimuth resolution $\delta_{a z}=L_{R x} / 2 N$ ) leads to $N=4$ of azimuth channels.

The performance parameters of the system are quite good, however, such a system can practically not be realized due to the tremendous antenna length.

\subsection{Single Swath Sub-Pulse Mode}

A possibility to reduce the antenna length of the previous system to approximately half is to use sub-pulse techniques [7]. The basic idea is to illuminate the wide swath by two subpulses from two different azimuth positions by using two Tx antennas. Adding the second Tx antenna [8, 9] allows the system to be operated such that two pulses are transmitted within each Pulse Repetition Interval (PRI) where typically each sub-pulse is delayed by a small fraction of the PRI with respect to previous one as shown in Fig. 3.

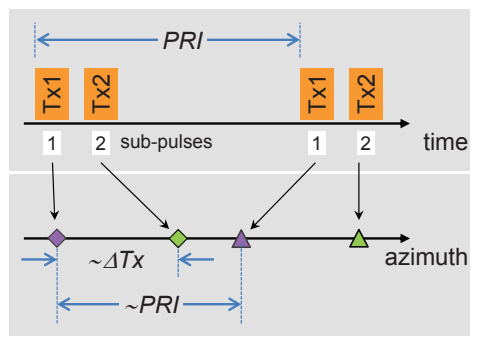

Fig. 3. Two sub-pulses showing that the position of the spatial samples depends on the Tx antenna separation and the PRI.

Doubling the number of Tx pulses within the same time interval consumes twice the power [7], but the number of received samples per PRI (i.e. the spatial sampling) is doubled which, here, allows reducing the $\mathrm{Rx}$ antenna length to half the values given by (2), i.e. $L_{R x}=L_{R x}^{\text {unif }} / 2=17.4 \mathrm{~m}$. The spatial distance between the azimuth samples of the two sup-pulses is mainly determined by phase center separation of the Tx antennas. Uniform sampling is achieved when $\Delta_{T x}=L_{R x} / 2$, which, for a single Tx/Rx antenna aperture, requires that the length of each Tx antenna be $L_{T x}=L_{R x} / 2=8.7 \mathrm{~m}$.

The echo signals of the two sub-pulses arrive at nearly the same time at the receiver. To separate the two echoes multi-SCORE is utilized here, where two receive beams are generated, each one maximized to the direction of arrival of one sub-pulse while suppressing the energy of the other $[8,9]$. The Rx antenna height $H_{R x}$ required to place a pattern null at the interfering sup-pulse is computed according to [3]:

$$
H_{R x}=\frac{2 \lambda r \tan \eta_{i}}{c_{0} \Delta \tau}
$$


where $r$ is the slant range and $\Delta \tau$ is the time delay between the the start of the two sub-pulses, respectively. Taking a single sub-pulse duty cycle of $6 \%$ gives $H_{R x}=13.4 \mathrm{~m}$ necessary to suppress the pulses at far range (worst case).

The SAR performance is shown in Fig. 4 in terms of the NESZ and Doppler pattern. The azimuth performance is comparable to the previous single-swath stripmap system, although here a total of 3 azimuth channels are used (effectively increasing the azimuth sampling) to compensate for the shorter Tx antenna length.

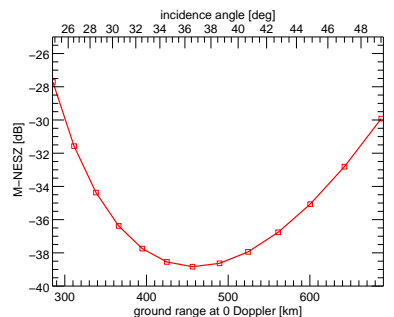

(a) NESZ

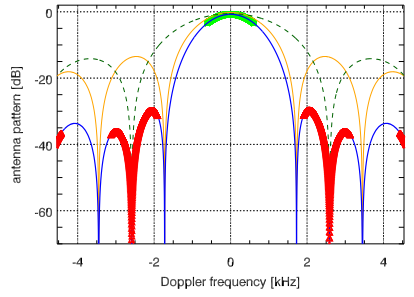

(b) Azimuth Pattern
Fig. 4. Noise-equivalent sigma-zero and azimuth pattern for single swath stripmap system utilizing sub-pulses.

\subsection{Multi-Azimuth ScanSAR}

Alternatively the ScanSAR burst mode may be used to cover the wide swath while utilizing multiple azimuth channels (MACs) for the required azimuth resolution [10], as has been suggested within the Sentinel-1 follow-on Study [11]. Here, a design is presented which results in an Rx antenna dimension of $12.2 \mathrm{~m} \times 2.8 \mathrm{~m}$ and 8 azimuth channels. As shown in the timing diagram of Fig. 5a the system is operated in $n_{\text {burst }}=4$ bursts, which is the minimum number needed if nadir echoes are taken into account by the timing considerations.

Fig. 5b shows that the NESZ variation within the swath is smaller compared to the previous modes, which is a result of the reduced sub-swath width. However, there is a Doppler dependent variation (scalloping) which is indicated by the different curves. The range performance is acceptable but for the extreme far range of the swath; nevertheless it is believed that a pattern optimization can yield the required performance, i.e. the antenna height is sufficient to allow for ambiguity suppression.

The burst mode operation has some advantages. Since only part of the swath is illuminated for each burst, the power density can be increased taking advantage of the high gain Tx antenna (assuming a Tx/Rx antenna configuration with TRM), which more or less compensates the reduced power density due to the wider illumination in azimuth. For the same reason the PRF can be increased and by this the total antenna

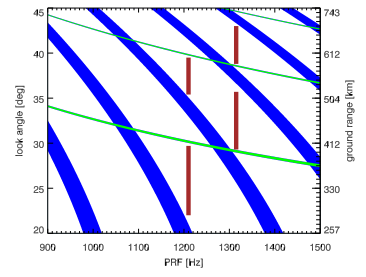

(a) Timing Diagram

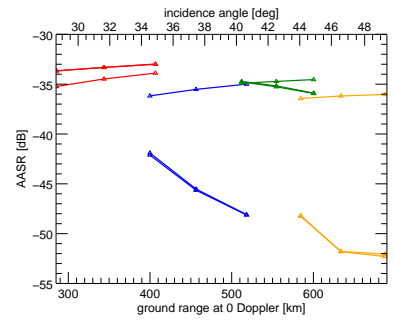

(c) AASR

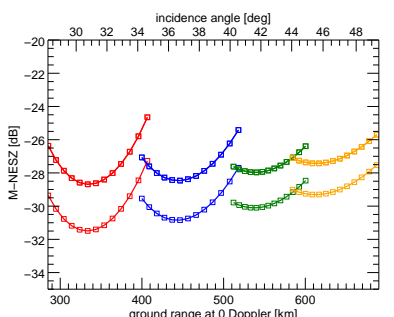

(b) NESZ

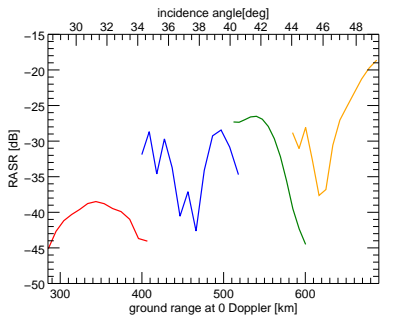

(d) RASR
Fig. 5. Timing diagram and SAR performance of the 4-burst ScanSAR MACs system.

length reduced. The ScanSAR mode is suitable for interferometric applications [12]. The disadvantage is somehow the required increase of the covered Doppler bandwidth by a factor of $n_{\text {burst }}+1$, which at the end results in a reduced $\mathrm{Rx}$ antenna gain due to the larger azimuth beamwidth. In total, the ScanSAR mode has an acceptable system efficiency.

\subsection{Multi-Azimuth Multi-Elevation Two Burst ScanSAR}

An alternative technique to cover a wide swath is the twoburst ScanSAR mode. Here multiple sub-swathes are imaged simultaneously taking advantage of DBF on-receive. To fill the gaps occurring due to the transmit instances, a burst mode operation is utilized, for which two PRFs/bursts are sufficient as seen in Fig. 6a.

Here the receive antenna dimensions are chosen to be $6.8 \mathrm{~m} \times 5.5 \mathrm{~m}$. This mode gives some flexibility in deciding on the antenna dimensions as long as the minimum antenna area does not go below a critical value. Here, the number of azimuth channels is $N=3$, which is determined by the length of the antenna element necessary to cover the system Doppler bandwidth.

The azimuth performance, cf. Fig. 6b, is good, mainly due to the oversampling effect. It is seen that the even numbered sub-swathes have a worse performance; this a result of the PRF values (cf. (2)), which result in a $3 \%$ non-uniform azimuth sampling [13]. The elevation performance (RASR) does not satisfy the requirement, as seen from Fig. 6c. Nevertheless, the antenna height of $5.5 \mathrm{~m}$ is considered sufficient for ambiguity suppression if a dedicated beam-forming tech- 


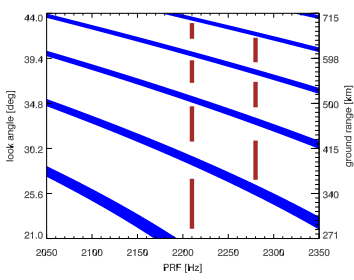

(a) Timing Diagram

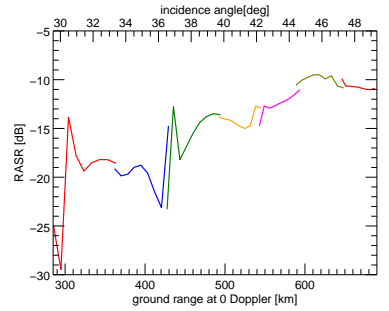

(c) AASR

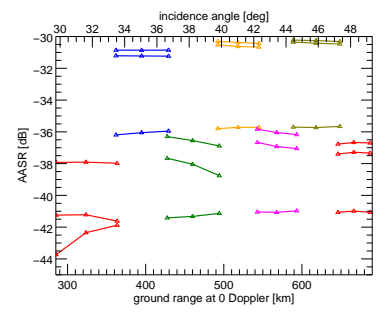

(b) NESZ

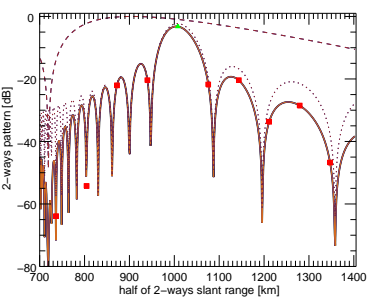

(d) RASR
Fig. 6. Timing diagram and SAR performance of the 2-burst ScanSAR MACs system.

nique is used. From Fig. 6d it is seen that the angular separation between the pattern nulls is such that they could be positioned at the two nearest ambiguities.

The advantage of this mode is that it needs only two bursts. On the other hand there are two main disadvantages which reduce the system efficiency. One is that the number of channels is higher than what is needed to reconstruct the instantaneous Doppler bandwidth, which effectively increases the data rate. The second disadvantage is that the transmit beam illuminates the complete swath, although only part of it (approximately the half) is imaged for each burst; this increases the required power.

\section{CONCLUSION}

The paper presented various techniques suitable for multichannel SAR systems. The SAR performance of these modes were shown for an exemplary L-band SAR required to image a $400 \mathrm{~km}$ swath. The main purpose was to show how the trade-space parameters of multi-channel SAR can be utilized and to explain their impact on the system design.

\section{REFERENCES}

[1] Nico Gebert, Multi-Channel Azimuth Processing for HighResolution Wide-Swath SAR Imaging, Ph.D. thesis, German Aerospace Center (DLR), Aug. 2009.

[2] Marwan Younis, Digital Beam-Forming for High Resolution Wide Swath Real and Synthetic Aperture Radar, Ph.D. thesis, Institut für Höchstfrequenztechnik und Elektronik, Universität Karlsruhe, July 2004.
[3] Gerhard Krieger, Nicolas Gebert, and Alberto Moreira, "Multidimensional waveform encoding: A new digital beamforming technique for synthetic aperture radar remote sensing," IEEE Transactions on Geoscience and Remote Sensing, vol. 46, no. 1, pp. 31-46, Jan. 2008.

[4] Christian Fischer, Christoph Schaefer, and Christoph Heer, "Technology development for the HRWS (High Resolution Wide Swath) SAR," in International Radar Symposium IRS'07, Sept. 2007.

[5] Glen D. Callaghan, Wide-Swath Space-Borne SAR: Overcoming the Trade-Off between Swath-Width and Resolution, Ph.D. thesis, University of Queensland's, 1999.

[6] Martin Süß and Werner Wiesbeck, "Side looking SAR system," Mar. 2005.

[7] Marwan Younis, Paco López-Dekker, Federica Bordoni, Piotr Laskowski, and Gerhard Krieger, "Exploring the trade-space of MIMO SAR," in Proc. Int. Geoscience and Remote Sensing Symposium IGARSS'13, Melbourne, Australia, July 2013.

[8] Paco López-Dekker, Marwan Younis, Jose A. García, Thomas Börner, and Gerhard Krieger, "Advanced digital beamforming architectures and operation modes for an enhanced SIGNAL mission concepts," in Proc. 1st workshop on Ka-band Earth Observation Radar Missions (KEO'12), Noordwijk, The Netherlands, Nov. 2012.

[9] Marwan Younis, Paco López-Dekker, Anton Patyuchenko, and Gerhard Krieger, "Digital beamforming architecture and techniques for a spaceborne interferometric ka-band mission," in Proceedings of the IEEE Radar Conference, Ottawa, Canada, Apr. 2013.

[10] Nicolas Gebert, Gerhard Krieger, and Alberto Moreira, "Multichannel ScanSAR for high-resolution ultra-wide-swath imaging," in Proc. European Conference on Synthetic Aperture Radar EUSAR'08, Friedrichshafen, Germany, June 2008.

[11] ESA, "Integrated tile demonstrator," Phase 1, Final Report, ESA contract no. 4000103316, European Space Agency ESA/ESTEC, 2013.

[12] Pau Prats-Iraola, Nestor Yague-Martinez, Steffen Wollstadt, Thomas Kraus, and Rolf Scheiber, "Demonstration of the applicability of 2-look burst modes in non-stationary scenarios with TerraSAR-X," in Proc. European Conference on Synthetic Aperture Radar EUSAR'2016, Hamburg, Germany, June 2016.

[13] Gerhard Krieger, Nicolas Gebert, and Alberto Moreira, "Unambiguous SAR signal reconstruction from non-uniform displaced phase centre sampling," IEEE Geoscience and Remote Sensing Letters, vol. 1, no. 4, pp. 260-264, Oct. 2004. 